\title{
Demyelinating lesions as a presenting feature of pernicious anaemia: Case report and literature review
}

\author{
Rahul Daimari ${ }^{1}$, Anthony A Oyekunle*1, Cassandra A Ocampo ${ }^{1}$, Lawrence Kwape ${ }^{2}$ \\ ${ }^{1}$ Department of Internal Medicine, Faculty of Medicine, University of Botswana, Gaborone, Botswana \\ ${ }^{2}$ Department of Internal Medicine, Princess Marina Hospital, Gaborone, Botswana
}

Received: March 19, 2017

Accepted: May 24, 2017

Online Published: June 6, 2017

DOI: $10.5430 /$ crim.v4n3p7

URL: https://doi.org/10.5430/crim.v4n3p7

\begin{abstract}
Background: Pernicious anaemia (PA) describes megaloblastic anaemia resulting from cobalamin deficiency that is due to the absence of intrinsic factor (IF). Most cases are autoimmune in origin, with antibodies to parietal cells, IF or the cobalamin - IF complex.

Methods: We report the clinical features, investigation and treatment of a patient in whom the first presentation of PA was demyelinating brain lesions. The patient presented with clinical features initially of neurological impairment and subsequently anaemia. Imaging studies were consistent with demyelinating lesions extending from the cortex to the midbrain. Peripheral blood and bone marrow findings were consistent with megaloblastic anaemia, which were confirmed by subnormal serum cobalamin levels. The patient was treated with parenteral cobalamin and oral folic acid.

Results: The patient responded with complete resolution of anaemia and complete clinical neurological response.

Conclusion: Clinical, laboratory and radiologic findings are important in the screening of patients presenting with demyelinating lesions, as these may help in the diagnosis of rare cases of PA. These tests are just as relevant even in the young African female population.
\end{abstract}

Key Words: Cobalamin, Pernicious anaemia, Demyelination

\section{INTRODUCTION}

Historically, before the discovery of cobalamin, the term pernicious anaemia (PA) was used to describe a progressive and usually fatal anaemia that was unresponsive to iron and blood transfusions. However, in recent times, the term has been restricted to describe megaloblastic anaemia resulting from cobalamin deficiency that is due to autoimmune atrophic gastritis affecting the production or function of intrinsic factor (IF).

Classical PA usually involves autoantibodies to IF or specific membrane antigen on parietal cells or both; with associated diffuse destruction of the body-fundus mucosa of the stomach and which leads to progressive loss of gastric glands, parietal cells and chief cells. The hypochlorhydria or achlorhydria which arise from severe parietal cell loss is responsible for the consequent hyperplasia of gastrin-producing G-cells in the antral mucosa. This eventually leads to increased gastrinemia, which stimulates hyperplasia of enterochromaffin-like (ECL) cells in the gastric body. Terminally, atrophic gastritis ensues.

Intrinsic factor is a $60 \mathrm{kDa}$ glycoprotein that strongly binds dietary cobalamin, forming the cobalamin-IF complex which

\footnotetext{
*Correspondence: Anthony A Oyekunle; Email: oyekunleaa@yahoo.co.uk; Address: Department of Internal Medicine, Faculty of Medicine, University of Botswana, Gaborone, Botswana.
} 
is absorbed in the terminal ileum, after binding to IF receptors on the luminal membranes of ileal cells. ${ }^{[1]}$ Two principal mechanisms are responsible for PA. First, the progressive destruction and eventual loss of parietal cells leading to failure of IF production. Indeed, the severity of the gastric lesion correlates with the degree of impaired IF secretion and the reduction in cobalamin absorption. ${ }^{[2]}$ Second, blocking autoantibodies occupy the cobalamin-binding site of IF, thereby preventing the formation of the cobalamin-IF complex. ${ }^{[3]}$

Cobalamin is required for DNA synthesis, and its deficiency perturbs the most rapidly dividing tissues of the bone marrow and gastrointestinal mucosa.

Pernicious anaemia is believed to account for as much as $80 \%$ of megaloblastic anaemia in the USA. A relatively recent survey in the USA found that $1.9 \%$ of elderly persons (> 60 years old) have undiagnosed PA. ${ }^{[4]}$ Incidence of PA is estimated to be $120 / 100,000$ in the UK, with a prevalence of up to $2 \%-3 \%$ in the Western countries and a median age at diagnosis of 60 years. PA has been described in all ethnic groups, though it is reported to be more common among women than men. ${ }^{[5]}$

The onset and progression of PA are slow. The usual presentation of PA is with symptoms of anaemia; though asymptomatic patients are frequently identified from routine hematologic investigations.

Peripheral blood findings typically include the presence of erythrocyte macro-ovalocytes and hypersegmented neu- trophils. The bone marrow characteristically shows megaloblastic erythroid precursors, with the classical nucleocytoplasmic developmental asynchrony.

The classically described neurological manifestation of severe cobalamin deficiency is subacute combined degeneration of the spinal cord and peripheral polyneuropathy. Early signs include the loss of vibratory and joint position sensations. Isolated peripheral neuropathy is less frequent but possibly underreported. Optic neuropathy occasionally occurs in adult patients with cobalamin deficiency, and it is characterised by symmetric, painless, progressive visual loss. Cobalamin deficiency has been linked to cognitive dysfunction in elderly subjects. Symptoms include slow mentation, memory impairment, attention deficits and dementia. Psychosis may also be the presenting symptom of cobalamin deficiency. Reported symptoms include suspiciousness, persecutory or religious delusions, auditory and visual hallucinations and disorganised thought processes. ${ }^{[6,7]}$

The most common MRI finding is increased symmetrical T2-weighted signal intensity confined to the posterior or posterolateral columns, and worse in the cervical and thoracic spinal cord. Confluent areas of abnormal signal intensity on T2-weighted images have been described in the cerebral white matter. Contrast enhancement of these lesions are uncommon and if present, mild. Following treatment for cobalamin deficiency, there is interval improvement of signal abnormality. Resolution of white matter changes are often seen within a few months of starting cobalamin therapy.
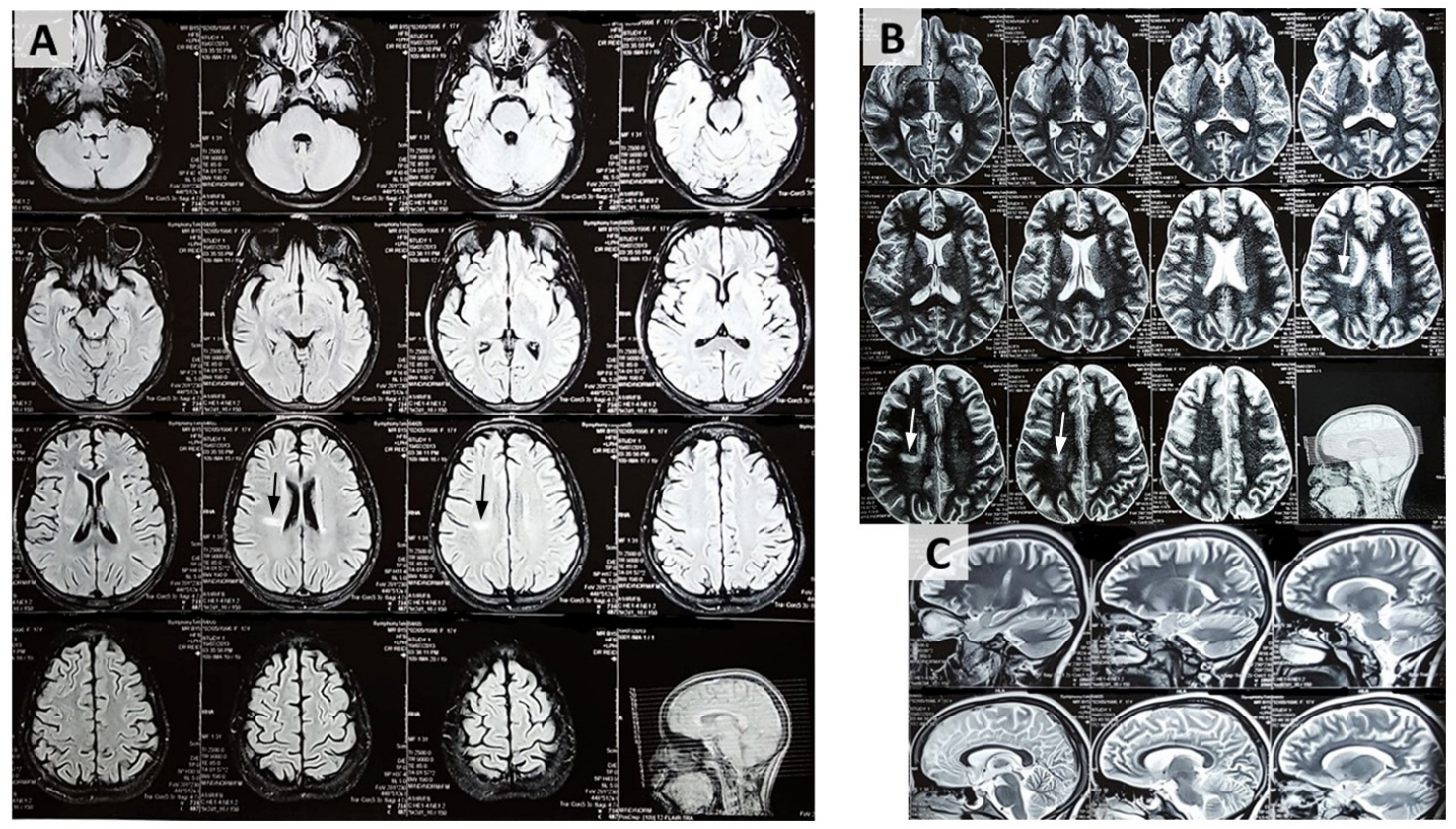

Figure 1. Flair (A) and T2-weighted (B \& C) MRI images, showing demyelinating lesions in the brain (shown with dark and light arrows) 
Pernicious anaemia is characteristically diagnosed by the presence of serum antibodies against IF and parietal cells. The IF antibody has high specificity with high positive predictive value (95\%) for the presence of PA. It is rarely found in conditions other than PA, and it identifies those requiring lifelong cobalamin replacement therapy. Unfortunately, sensitivity is reported to be as low as $35 \%-55 \%$. Similarly, in up to $80 \%$ of patients with PA, IF antibodies are detectable in gastric juice.

Though there is no consensus on cobalamin replacement therapy, most experts will give intramuscular cobalamin $1 \mathrm{mg}$ injections daily for 1-2 weeks, weekly for one month, and monthly for life. The administration of folate without cobalamin has been associated, in some patients, with worsening of neurologic dysfunction, and is consequently discouraged.
Additionally, cobalamin deficiency-induced neurologic dysfunction, quite unlike hematologic defects, may not always reverse despite adequate cobalamin replacement.

\section{CASE PRESEntation}

Miss ML, a 19-year-old HIV-negative lady, was admitted on account of symptomatic anaemia, for which she had received multiple blood transfusions over the last four years. During that admission, she had left-sided upper limb weakness, with her brain MRI showing T2 and FLAIR non-enhancing lesions mostly in the right subcortical areas - internal capsule, midbrain, cerebral peduncle and frontal subcortical white matter; highly suggestive of demyelination (see Figure 1). The patient's current presentation was for a one-month history of easy fatigability and dizzy spells. Examination revealed essentially marked conjunctival pallor and no organomegaly.
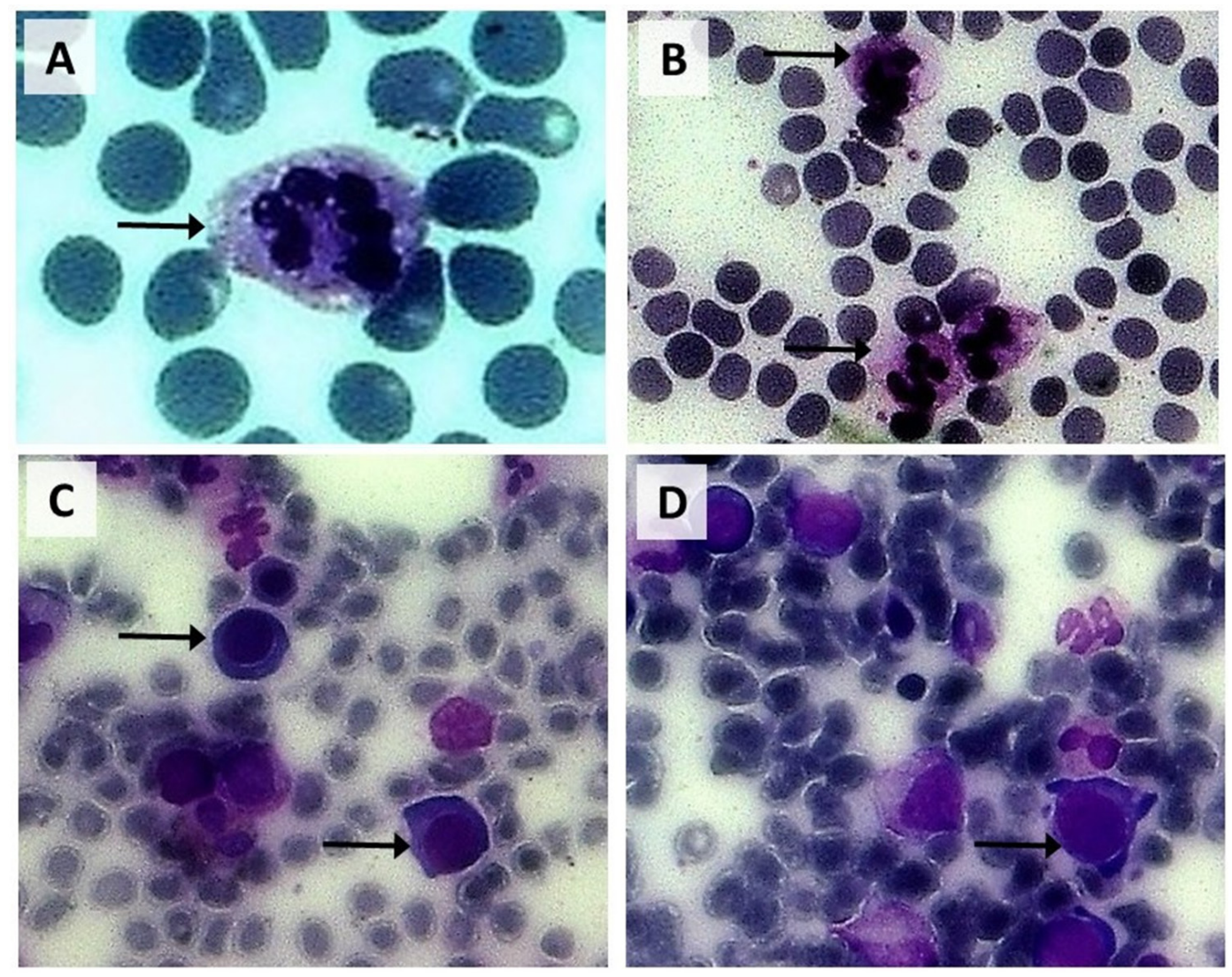

Figure 2. Peripheral blood smears (A and B) showing hypersegmented neutrophils and red cell macro-ovalocytosis. Bone marrow smears (C and $\mathrm{D})$ shows multiple megaloblastic normoblasts.

Blood tests revealed severe anaemia (haemoglobin $[\mathrm{Hb}]$ $3.5 \mathrm{~g} / \mathrm{dl})$, moderate thrombocytopenia $\left(70 \times 10^{9} / \mathrm{L}\right)$, mild predominantly unconjugated hyperbilirubinemia (total bilirubin $28.7 \mathrm{mmol} / \mathrm{L}$, conjugated bilirubin $5.4 \mathrm{mmol} / \mathrm{L}$ ), and high serum lactate dehydrogenase 5,158 U/L. Peripheral blood smear showed marked anisocytosis, macro-ovalocytosis, and numerous hypersegmented neutrophils. Bone marrow aspirate showed erythroid hyperplasia with megaloblastic erythroid precursors (see Figure 2). Serum folate was normal, while cobalamin was very low $(<61 \mathrm{pmol} / \mathrm{L})$, and she was found to be positive for both anti-parietal cell and anti-IF antibodies. 
The patient was commenced on parenteral cobalamin (and oral folate), with prompt resolution of anaemia, and no further need for blood transfusion.

\section{Discussion}

Cobalamin deficiency is a major epidemiologic concern in the Indian subcontinent, Mexico, Central and South America. ${ }^{[8]}$ It is commonly seen among strict vegetarians. Pernicious anaemia is a well-recognised cause of non-dietary cobalamin deficiency, and it is a common cause of megaloblastic anaemia worldwide. Ancient literature suggests that PA is less common among blacks, though with improved awareness, the disease is being increasingly diagnosed among African populations. ${ }^{[8]}$

In our patient, there was anaemia, megaloblastic picture in peripheral smear and bone marrow, positive anti-IF and antiparietal cell antibodies; findings diagnostic of PA.

Our patient, a young Batswana lady, had an early-onset neurological impairment, severe anaemia and anti-IF antibodies. A recent study comparing black Americans with South Africans had found that $14 \%$ of the PA patients were $<30$ years, and the authors had suggested that there may be an earlier age of onset of amongst blacks than the reported age incidence in whites. The same study also noted that there was a significantly higher incidence of the anti-IF antibodies among women compared to men. ${ }^{[10]}$ It is equally interesting that the neurologic features were initially more prominent than the anaemia. This may have been due to attempts to correct anaemia with oral hematinics including folate, which would not correct the neurologic features. Similarly, lack of correlation between neurologic defects and anaemia has been previously reported by some researchers. ${ }^{[9]}$

Patients with cobalamin deficiency may also present with neuropsychiatric features such as dementia, depression, acute psychosis and cerebrovascular disease. It may be subtle and less evident, and only a high index of suspicion may prompt the physician to undertake cobalamin testing.

This patient had also presented with radiologic features of unilateral demyelination extending from the cortex to the midbrain. Cobalamin deficiency is known to cause intramedullary lesion, mainly symmetric myelin loss in the posterior and lateral column, though patients have been known to develop lateralising signs and can progress to hemiplegia, due to asymmetric demyelination of the cord. Unilateral demyelination is uncommon, though it has been described in association with both megaloblastic anaemia and multiple sclerosis (MS). Indeed, Reynolds et al. have recently reported ten patients with both conditions, with the authors suggesting that their coexistence may be more than coincidental. ${ }^{[11]}$ Cobalamin deficiency results in defective myelin synthesis, leading to central and peripheral nervous system dysfunctions.

It is noteworthy that the neurologic symptoms preceded the hematologic features. Similar neuro-haematologic dissociations have been previously reported by others. ${ }^{[12]}$ Similarly, demyelination involving the brain but not the spinal cord has been reported in patients with cobalamin deficiency. In these patients, hyperintensity signals are typically restricted to the subcortical and the periventricular white matter.

In this case, amyotrophic lateral sclerosis can be excluded mostly on the grounds of the rather short presentation, though it is also uncommon in the young and rarely presents with features of megaloblastic anaemia. A gastric biopsy would have been helpful to establish the presence of atrophic gastritis, an invariable finding in PA, but this could not be done.

\section{Conclusion}

Clinical, laboratory and radiologic findings are important in the screening of patients presenting with demyelinating lesions, as these may help in the diagnosis of rare cases of PA. These tests are just as relevant even in the young African female population. Early diagnosis is essential, as it allows for starting replacement therapy and avoiding irreversible neurological damage.

\section{CONFlicts OF INTEREST Disclosure}

The authors have declared no conflicts of interest.

\section{REFERENCES}

[1] Seetharam B, Alpers D, Allen R. Isolation and characterization of the ileal receptor for intrinsic factor-cobalamin. J Biol Chem. 1981[cited 2017Mar5]. Available from: https://www.researchgate.net/profile/David_Alp ers/publication/16935056_Isolation_and_characteriz ation_of_the_ileal_receptor_for_intrinsic_factor-c obalamin/links/53f60ac40cf2888a749251e4.pdf

[2] Wickramasinghe SN. Diagnosis of megaloblastic anaemias. Blood
Rev. 2006 Nov; 20: 299-318. PMid:16716475 https : //doi .org/ 10.1016/j.blre.2006.02.002

[3] Toh BH, van Driel IR, Gleeson PA. Pernicious Anemia. N Engl J Med. 1997 Nov 13; 337: 1441-8. PMid:9358143 https ://doi .or g/10.1056/NEJM199711133372007

[4] Carmel R. Prevalence of Undiagnosed Pernicious Anemia in the Elderly. Arch Intern Med. 1996 May 27; 156: 1097. PMid:8638997 ht tps://doi.org/10.1001/archinte.1996.00040041097008

[5] Hoffbrand AV, Higgs DR, Keeling D, et al. Postgraduate haematology. 
[cited 2017 Mar 5]. Available from: https://books.google.co. bw/books?hl=en\&lr=\&id=AvhcCgAAQBAJ\&oi=fnd\&pg=PR7\&d $q=$ Hof $f$ brand+Postgraduate+Hematology\&ots $=8 \mathrm{sbhDLBi} 9$ K\&sig=e047Cg1nFtQRuvf-31SjN6Cqrm8\&redir_esc=y\#v=0 nepage\& $q=$ Hof $f$ brandPostgraduateHematology $\& f=f a l$ se

[6] Hector M, Burton JR. What are the Psychiatric Manifestations of Vitamin B 12 Deficiency? J Am Geriatr Soc. 1988 Dec; 36: 1105-12. PMid:3057051 https://doi .org/10.1111/j.1532-5 415.1988.tb04397.x

[7] Berry N, Sagar R, Tripathi BM. Catatonia and other psychiatric symptoms with vitamin B12 deficiency. Acta Psychiatr Scand. 2003 Aug; 108: 156-9. PMid:12823174 https://doi.org/10.1034/j.16 00-0447.2003.00089.x

[8] Stabler SP, Allen RH. Vitamin B12 deficiency as a worldwide problem. Annu Rev Nutr. 2004 Jul 14; 24: 299-326. PMid:15189123 http $\mathrm{s}$ ://doi.org/10.1146/annurev.nutr.24.012003.132440
[9] Solanki DL, Jacobson RJ, Green R, et al. Pernicious Anemia in Blacks: A Study of 64 Patients from Washington, D. C, and Johannesburg, South Africa. Am J Clin Pathol. 1981 Jan 1; 75: 96-9. PMid:7457436 https ://doi.org/10.1093/ajcp/75.1.96

[10] Ralapanawa DMP, Jayawickreme KP, Ekanayake EMM, et al. B12 deficiency with neurological manifestations in the absence of anaemia BMC Res Notes. 2015 Dec 18; 8: 458. PMid:26385097 https: //doi.org/10.1186/s13104-015-1437-9

[11] Reynolds EH, Linnell JC, Faludy JE. Multiple Sclerosis Associated With Vitamin B12 Deficiency. Arch Neurol. 1991 Aug 1; 48: 80811. PMid:1898255 https://doi.org/10.1001/archneur.199 1.00530200044017

[12] Briani C, Torre C, Citton V, et al. Cobalamin Deficiency: Clinical Picture and Radiological Findings. Nutrients. 2013 Nov 15; 5: 4521-39. PMid:24248213 https://doi.org/10.3390/nu5114521 